\title{
Editorial
}

\section{Data: Infinity in a grain of sand}

Journal of Direct, Data and Digital Marketing Practice (2015) 17, 1. doi:10.1057/dddmp.2015.39

If you have been to the beach on your summer holidays this year, chances are you may have returned with a few grains of sand still clinging somewhere. One of the remarkable aspects of sand is just how much attention a single grain can demand (although exactly where you find it is a big part of that...)

Data has become like sand. For many organizations, it starts as a single point of irritation - an error or missing variable that gets in the way of a core business process. Start to fix that problem and it leads you deeper into the sand dunes - piles of data that you discover have been piled up across the business, often ignored or considered to be of little value. At some point, you may experience the data epiphany - from a single grain of truth you can gain access to an infinity of wisdom. Data is restless, like the desert. That movement is what makes data management so challenging and why data can be exploited as an almost limitless resource. Everything that happens in the organization sweeps in more data, offers new opportunities and creates fresh problems.

In this special issue of the Journal, assembled with the invaluable input of the IDM Data Council, you can discover something of that breadth and depth of data. In the whitepaper by Jiyao Xun, 'Return on website visit duration', one of the most common data sets - clickstream and web analytics — is examined afresh for its potential. In Mardien Drew and Paul Berney's opinion piece, 'What does mobile really mean for research?', they consider how the new data streams pouring out of consumer interactions via the mobile phone create new ways to gain insight into behaviour and intentions. Virtually every business has a website and most now offer an app or mobile-optimized access, ensuring that they too can tap into these new marketing techniques.

Two risks are also highlighted within this issue. The first comes from both new data protection legislation and potential consumer pushback against the extent to which their personal information is being harvested for corporate benefit, rather than their own. The second comes from organizations getting so wrapped up in their digital transformation and the data sandbanks piling up as a result that they forget about the human touch and making an emotional connection with customers.

These are timely cautions at a time when the rush to extract ever more value from data is in full swing. Marketers have always understood that their relationship with customers is a two-way thing and that access to those individuals is a privilege (or at least they should have). If that understanding gets lost in the infinity of data being analysed, marketers may find themselves being rubbed sore by a single grain they have overlooked, yet which could spill the whole desert into their office. 